\title{
Investigation the Effect of Octreotide and Pituitrin on Upper Gastrointestinal Bleeding in Cirrhosis
}

\author{
Min Feng \\ Shandong Zibo City Center Hospital Digestion in a Family, Zibo, \\ Shandong, 255036
}

\begin{abstract}
Cirrhosis of the esophagus in the stomach of the vein prone is to variceal bleeding and bleeding, which is the most common gastrointestinal bleeding. Cirrhosis of the digestive tract bleeding is an acute and severe internal medicine, one of its rapid onset, rapid progress, large amount of bleeding, serious harm to the lives of patients, it should be early treatment. It is reported that early effective control of patients with gastrointestinal bleeding can significantly reduce mortality. To this end, the use of octreotide in the treatment of liver cirrhosis patients with upper gastrointestinal bleeding, and the clinical efficacy of pituitrin compared to evaluate the effect of hemostasis.
\end{abstract}

Keywords: Octreotide, Pituitrin, Upper Gastrointestinal Bleeding

\section{Introduction}

Investigate the efficacy of octreotide and pituitrin in the treatment of upper gastrointestinal bleeding in patients with liver cirrhosis. Methods 62 patients with upper gastrointestinal hemorrhage from March 2009 to October 2009 in our hospital were randomly divided into two groups, 31 cases in each group. The control group was treated with pituitrin on the basis of basic therapy, In the control group based on the addition of octreotide treatment, a comparative analysis of the two groups of treatment. Results The total effective rate was $93.5 \%$ in the observation group and $71.0 \%$ in the control group, the difference was statistically significant $(\mathrm{p}<0.05)$. The adverse reaction rate was $16.1 \%$ in the 
observation group and $12.9 \%$ in the control group Statistical significance ( $\mathrm{p}>$ 0.05). Conclusion Octreotide and pituitrin in treatment of cirrhosis of the upper gastrointestinal bleeding patients have satisfactory results, and fewer adverse reactions, it is worthy of clinical application.

\section{Materials and methods}

A total of 62 patients with cirrhosis of the upper digestive tract were admitted to our hospital from March 2009 to October 2009, including 38 males and 24 females with an average age of 25 to 69 years (mean $43.2 \pm 3.9$ years) ), All patients had a clear hematemesis and (or) asphalt tarry stool and other upper gastrointestinal bleeding clinical manifestations, and blood, liver function, B ultrasound or CT, endoscopy and other tests diagnosed with cirrhosis and lower esophagus or Gastric variceal bleeding, excluding other diseases caused by bleeding. All patients were randomized into two groups of 31 patients. The observation group, 20 males and 11 females, aged 27 to 69 years, mean (43.7 \pm 3.6) years. The control group, 18 males and 13 females, aged 25 to 68 years, mean $(43.0 \pm 3.5)$ years. There was no significant difference between the two groups $(\mathrm{p}>0.05)$.

(2) effective, after 24 to 72 hours to stop bleeding within the treatment of bleeding after treatment, after treatment, the clinical symptoms disappeared, blood pressure and pulse recovery, hemoglobin returned to normal, blood urea nitrogen increased phenomenon disappeared; (3) is invalid, the patient is still 72 hours after hematemesis, melena and other clinical manifestations, blood pressure, pulse instability, hemoglobin levels continued to decline. Total effective rate total effective rate - (number of effective cases + effective cases) / total number of cases $\times 100 \%$.

Routine use of supplementary blood volume, liver, corrects electrolyte imbalance and other treatment. On the basis of this, the control group was treated with intravenous infusion of $10 \mathrm{u}$ of pituitrin and $40 \mathrm{ml}$ of normal saline for $15 \mathrm{~min}$, then infused with $12 \mathrm{U}$ into $250 \mathrm{ml}$ of $5 \%$ glucose injection, drip rate of $20 \sim 25$ drops / $\mathrm{min}, 1$, and then repeated every $4 \mathrm{~h}$ to give dose, $72 \mathrm{~h}$ reduced by half to maintain $48 \mathrm{~h}$. In the observation group, octreotide was added on the basis of the control group. Octreotide was infused intravenously at $100 \mu \mathrm{g} / \mathrm{h}$, followed by continuous infusion of $25 \mu \mathrm{g} / \mathrm{h}$ for $24-96 \mathrm{~h}$.

Effective: medication $48 \sim 72 \mathrm{~h}$ gastric tube suction (or blood stasis), the blood pressure and heart rate returned to normal and stable; effective: medication $48 \mathrm{~h}$ after the patient hematemesis and (or) blood in the stool stop, gastric tube suction fluid volume decreased significantly, Fluid to clear, blood pressure, heart rate continued to be stable; invalid: medication $72 \mathrm{~h}$ gastric tube aspirate is still bloody, blood pressure, heart rate instability, and switch to other treatment measures. Total efficiency $=$ significant efficiency + efficiency.

The SPSS11.0 statistical software package was used to analyze the count data. The results were analyzed by $\chi 2$ test, $\mathrm{p}<0.05$ was considered statistically significant. 


\section{Results Analysis}

The total effective rate was $93.5 \%$ in the observation group and $71.0 \%$ in the control group, the difference was statistically significant $(\chi 2=5.4153, p<0.05)$. Upper gastrointestinal bleeding is the most common complication of liver cirrhosis and high mortality, is more than the ligament of the digestive tract, including esophagus, stomach, duodenum or pancreas and other diseases caused by bleeding, gastrojejunostomy Of the jejunum lesion bleeding is also within this range. Cirrhosis of the upper gastrointestinal bleeding for several reasons [3-4]: (1) esophageal varices, esophagus, gastric varices is the leading cause of cirrhosis of the upper gastrointestinal bleeding the most important reason. Cirrhosis of the liver can lead to increased portal pressure, esophageal, gastric varices, when the portal vein or hepatic venous obstruction can exacerbate portal hypertension, leading to varicose esophagus, gastric variceal bleeding, causing upper gastrointestinal bleeding. (2) gastrointestinal mucosal erosion, cirrhosis in patients with portal hypertension, often resulting in gastrointestinal congestion, mucosal edema erosion, causing bleeding. (3) cirrhosis of portal hypertension in patients with peptic ulcer incidence higher than normal, the incidence rate of $8 \%$ to $20 \%$, and gastric ulcer is the cause of esophageal variceal rupture of the reasons, and gastric ulcer due to cirrhosis of the liver Cause, this reason is also attributed to cirrhosis of the digestive tract bleeding. (4) gastrointestinal failure, advanced liver cirrhosis due to liver dysfunction caused by gastrointestinal secretion, absorption, exercise, obstruction and circulation to force the dysfunction, known as hepatic and gastrointestinal dysfunction. In severe liver failure late, due to nutritional disorders, bacterial damage, mucosal barrier damage, gastric acid erosion, digestion and coagulation dysfunction and other reasons, can often see gastrointestinal mucosal erosion, ulcers, leading to gastrointestinal bleeding, also belong to cirrhosis of the liver Gastrointestinal bleeding one of the reasons. (5) antral capillary telangiectasia, cirrhosis of portal hypertension in patients with mucosal lesions mainly in the antrum (not in the bottom of the stomach) inherent capillary expansion of the superficial capillaries, gastroscope showed multiple red spots this situation Of the cirrhosis of the digestive tract is not common.

Upper gastrointestinal bleeding rescue principles are: supportive therapy, infusion, blood transfusion, in order to prevent and correct shock, including drug hemostasis, mechanical compression (three cavity two balloon tube compression) to stop bleeding, endovascular embolization to stop bleeding and surgical hemostasis. In this study, the use of drugs to stop bleeding, the main study octreotide and pituitrin treatment of cirrhosis of the upper gastrointestinal bleeding in patients with the efficacy of octreotide and pituitrin treatment group was significantly better than the control group, and the incidence of adverse reactions was not significant difference. Pituitrin is a vasoconstrictor, by contraction of visceral vascular bed of small arteries, reducing portal vein blood flow and pressure. Octreotide is a synthetic octapeptide ring-loaded compounds, with natural endogenous somatostatin similar to the role, but the role of strong and lasting half-life 30 times longer than natural inhibitory, which can inhibit 
gastric acid, gastrin And pepsin secretion, inhibition of gastric acid secretion and proton pump inhibitor similar; can improve gastric mucosal blood supply, the gastrointestinal mucosa has a protective effect, can promote mucosal repair; can selectively reduce the portal vein and collateral circulation Of blood flow and pressure, reduce the pressure of esophageal and gastric varices, inhibit glucagon secretion and antagonism of glucagon on visceral blood vessels of the expansion of the role of systemic hemodynamics.

Pituitrin is a strong contraction of blood vessels, a "medical hemostatic forceps," the name. It is by contraction of visceral vascular bed arteries and capillaries before the sphincter, reducing the portal vein blood flow, so that rupture thrombosis and achieve hemostasis purposes, is the treatment of upper gastrointestinal bleeding first-line drugs. However, due to poor selectivity, systemic hemodynamics have a certain impact, small doses can lead to heart, kidney, brain and other systemic hypoxia, intravenous infusion often appear high blood pressure, myocardial ischemia, abdominal pain, stool frequency Increased and other adverse reactions, severe cases can induce angina pectoris, myocardial infarction, for this reason in elderly patients, cardiovascular and cerebrovascular diseases in patients with limited application. Therefore, in the elimination of these adverse reactions, the need to add nitroglycerin, the two have a synergistic effect, the mechanism is as follows: it can dilate the vein and expansion of the arterial two ways, first, dilate the vein can reduce myocardial preload, Second, the expansion of the cerebral artery, coronary artery, gastrointestinal smooth muscle, in the common role, so that reduced portal vein blood flow, portal vein pressure decreased.

Octreotide is a synthetic octapeptide somatostatin analogue, half-life of 1.5 $2 \mathrm{~h}$, the role of strong and lasting, visceral blood vessels selectively against the role of glucagon, reducing the portal vein pressure, the odd vein blood flow, thereby reducing Varicose vein pressure to achieve the purpose of hemostasis; the same time, can also inhibit gastric acid, pepsin and gastrin secretion, by reducing the blood vessels to reduce visceral blood flow of sex hormones, in particular, reduce portal vein blood flow. Among them, the biggest feature is the noncontractile effects of cardiovascular and cerebrovascular, high safety, fewer adverse reactions, this selective effect is better than pituitrin causes. The results showed that 45 patients with cirrhosis of the upper gastrointestinal bleeding in patients with octreotide, the effect is better, hemostasis success rate of $75.0 \%$, the average time to hemostasis $(21 \pm 11) \mathrm{h}$, the incidence of adverse reactions was $8.3 \%$ The hemostasis rate and total effective rate of octreotide group were significantly higher than that of pituitrin group, and the time of hemostasis and the incidence of adverse reactions were significantly less than that of pituitrin group, which indicated that octreotide had a high rate of hemostasis, hemostasis and adverse reactions Less of the advantages, the most important thing is that it does not affect systemic hemodynamics, especially for weak constitution, coronary heart disease, myocardial infarction patients. 


\section{Conclusion}

Cirrhosis and upper gastrointestinal bleeding is one of the common acute severe internal medicine, the disease is rapid, severe cases dues to low blood volume shock and endanger the lives of patients, and thus in clinical emergency, rapid and correct choice of hemostatic drugs is a top priority. Reported in the literature, treatment of upper gastrointestinal bleeding, first choice is endoscopic interventional therapy, but in the majority of primary hospitals, it is by the medical equipment and doctors limit the level of technology, emergency endoscopic treatment can not be carried out, medical drug therapy is still the preferred method. Conventional medical treatment, such as fasting, bed rest, expansion, hemostatic and other treatment, although the clinical treatment is necessary, but not completely and effectively achieve the hemostatic effect. Octreotide is a safe and effective treatment of upper gastrointestinal bleeding one of the drugs, but more expensive, and pituitrin as traditional medicine, this study aims to observe the octreotide and pituitrin in the treatment of cirrhosis of the upper gastrointestinal bleeding Efficacy difference.

\section{References}

[1] Omeprazole combined with pituitrin and nitroglycerin in the treatment of cirrhosis with upper gastrointestinal bleeding in 30 cases. Zhan. Hainan Medical, 9(2), pp. 91-95, 2011

[2] octreotide treatment of hepatitis B cirrhosis and esophageal and gastric variceal hemorrhage clinical observation. Wang Xiuli. Contemporary Medicine, 8(1), pp. 20-25, 2009

[3] domestic octreotide treatment of non-varicose veins upper gastrointestinal bleeding: 46 cases of observation. Wu Yulun, Xie Chaohua, Chen Yinghua. New Medicine, 07(3), pp. 10-12, 2008

[4] octreotide and pituitrin combined treatment of cirrhosis and upper gastrointestinal bleeding. Ji G, Yan Zhengping, Chen Jun, Wang Xiaoqiu, Yu Weizhou, Tai Shaoli, 8(1), pp. 15-21, 2008.

[5] Pituitrin and somatostatin on the control of esophageal variceal bleeding contrast analysis. Journal of Modern Medicinal Chemistry, 11(2), pp. 18-21, 2007 